\title{
The functional significance of chronotropic incompetence during dobutamine stress test
}

A Elhendy, R T van Domburg, J J Bax, P R Nierop, M L Geleijnse, M M Ibrahim, J R T C Roelandt

\begin{abstract}
Objective-To investigate the functional significance of chronotropic incompetence during dobutamine stress echocardiography.

Patients and methods-The functional significance of chronotropic incompetence was evaluated during dobutamine stress echocardiography in 512 patients without $\beta$ blocker treatment who underwent dobutamine stress echocardiography (up to $40 \mu \mathrm{g} / \mathrm{kg} / \mathrm{min}$ ) and completed the protocol or reached the target heart rate. Mean (SD) age was 60 (12) years (313 men, 199 women). Chronotropic incompetence was defined as failure to achieve $85 \%$ of the maximum exercise heart rate predicted for age and sex (220 - age in men; 200 - age in women) at maximum dobutamine dose.
\end{abstract}

Results-Chronotropic incompetence occurred in 196 patients (38\%). Affected patients were significantly younger, more likely to be men (both $p<<0.001$ ) and smokers $(p<0.05)$, had a higher prevalence of previous myocardial infarction $(p<0.005)$ and resting wall motion abnormalities $(p<0.05)$, and had a lower resting heart rate $(\mathrm{p}<<0.001)$ and systolic blood pressure $(p<<0.001)$ than patients without chronotropic incompetence, but there was no difference in the overall prevalence of ischaemia and significant coronary artery disease. By multivariate analysis, independent predictors of chronotropic incompetence were a lower resting heart rate $(\mathrm{p}<<0.001)$, younger age $(\mathrm{p}<0.001)$, and male sex (p $<<0.001)$.

Conclusions-The relations among sex, age, and chronotropic incompetence show the need to titrate the dobutamine dose using specific data based on age and sex related heart rate responses to dobutamine rather than to an exercise stress test. Obtaining specific heart rate criteria is necessary to determine whether chronotropic incompetence represents a real failure to achieve a normal response or is the result of applying an inappropriate gold standard.

(Heart 1999;81:398-403)

Keywords: dobutamine; echocardiography; chronotropic incompetence

Correspondence

Dr A Elhendy,

Wilhelminapark 14, 2342

AG Oepstgeest, Netherlands.

Accepted for publication

2 September 1998

Chronotropic incompetence during exercise stress test has been recognised as a predictor of increased mortality and of coronary heart disease.$^{1-5}$ Pharmacological stress testing using high dose dobutamine results in an increase in heart rate and myocardial contractility ${ }^{6}$; the dobutamine stress test is therefore considered to be an exercise simulator suitable for evaluating patients with known or suspected coronary artery disease. Some patients, however, fail to reach an adequate heart rate despite the maximum dose of dobutamine, even in absence of concurrent $\beta$ blocker treatment. ${ }^{7}$ Despite the known association between chronotropic incompetence during exercise stress testing and the risk of coronary artery disease, the functional significance of the impaired chronotropic response to dobutamine has not been evaluated. The identification of variables associated with chronotropic incompetence is important in order to determine whether this pattern correlates with markers of poor prognosis, and for possible modifications to the dobutamine stress protocol ${ }^{8}$ to achieve the target heart rate and thus optimise the sensitivity of dobutamine stress echocardiography. ${ }^{9-11}$ In this study we investigated the clinical, haemodynamic, and echocardiographic variables that contribute to chronotropic incompetence during dopamine stress echocardiography in patients with limited exercise capacity who were referred for evaluation of myocardial ischaemia but who were not taking $\beta$ blocker drugs.

\section{Methods}

PATIENT POPULATION

The study population comprised consecutive patients with limited exercise capacity, referred to our imaging laboratory for evaluation of myocardial ischaemia by dopamine stress echocardiography. The patients fulfilled the following criteria:

- Completion of the maximal dobutamineatropine stress testing protocol or achievement of $85 \%$ of the maximum exercise heart rate predicted for age and $\operatorname{sex}^{12}(220$ - age (in years) in men; 200 - age in women)

- No $\beta$ blocker treatment

- The presence of sinus rhythm at baseline electrocardiogram.

By these criteria, patients in whom the test was terminated prematurely before reaching the target heart rate or the maximum dobutamine-atropine dose owing to symptoms or side effects were not included in the study. There were 512 patients who fulfilled these criteria, mean (SD) age was 60 (12) years (313 
verbal informed consent to undergo the study. The hospital ethics committee approved the use of dopamine stress echocardiography for evaluation of patients with known or suspected coronary artery disease.

\section{DOBUTAMINE STRESS TEST}

Increasing doses of dobutamine were infused through an antecubital vein in three minute stages. The starting dose was $5 \mu \mathrm{g} / \mathrm{kg} / \mathrm{min}$, followed by $10 \mu \mathrm{g} / \mathrm{kg} / \mathrm{min}$, and increasing by 10 $\mu \mathrm{g} / \mathrm{kg} / \mathrm{min}$ every three minutes to a maximum of $40 \mu \mathrm{g} / \mathrm{kg} / \mathrm{min}$. Atropine (up to $1 \mathrm{mg}$ ) was given to patients not achieving $85 \%$ of maximum heart rate predicted for age and sex at the maximum dose of dobutamine, and the dobutamine infusion was continued. The ECG was monitored throughout the dobutamine infusion and recorded each minute. Cuff blood pressure was measured at rest, every three minutes during stress, and at maximum stress. The test was interrupted if there was severe chest pain, ST segment depression $>2 \mathrm{~mm}$, significant ventricular or supraventricular arrhythmia, hypertension (blood pressure $\geqslant 240$ / $120 \mathrm{~mm} \mathrm{Hg}$ ), systolic blood pressure fall of $>40 \mathrm{~mm} \mathrm{Hg}$, or any intolerable side effect regarded as being due to dobutamine. Metoprolol (1-5 mg) was used intravenously to reverse the effects of dobutamine if they persisted.

The test was considered successful if the patient could achieve $85 \%$ of the maximum heart rate or when there was an ischaemic end point (angina, ST segment depression, new or worsened wall motion abnormalities), or both. The selection criteria for the study determined that the end point of the test in all patients was the achievement of target heart rate or the administration of the maximum allowable dobutamine-atropine dose. Chronotropic incompetence was defined as the inability to reach $85 \%$ of the maximum heart rate predicted for age and sex at the maximum dose of dobutamine ( $40 \mu \mathrm{g} / \mathrm{kg} / \mathrm{min})$, as used in exercise studies. ${ }^{12}$ This cut off is also used in most laboratories to determine the need for atropine administration in patients with submaximal heart rate at peak dobutamine dose. ${ }^{13-17}$

\section{STRESS ECHOCARDIOGRAPHY}

Echocardiographic images were acquired at rest and during stress and on recovery. The echocardiograms were recorded on video tapes and were also digitised on optical disk and displayed side by side in quad-screen format (Vingmed CFM 800, Vingmed Sound A/S, Horten, Norway) to facilitate the comparison of rest and stress images. The left ventricular wall was divided into 16 segments and scored using a four point scale, where $1=$ normal, $2=$ hypokinesis, $3=$ akinesis, and $4=$ dyskinesis. Wall motion score index was derived by dividing the summation of individual score of the 16 segments by 16 . The interpretation of images was performed by two experienced observers without knowledge of the patients' clinical data. In case of disagreement, a majority decision was achieved by a third observer. In our laboratory, the inter- and intraobserver agreement for dobutamine stress echocardiography assessment is $92 \%$ and $94 \%$, respectively. ${ }^{18}$ Ischaemia was defined as new or worsening wall motion abnormalities. If segments that were akinetic at rest became dyskinetic during stress, without improvement with low dose dobutamine, this was not considered to reflect ischaemia, as previously reported. ${ }^{19}$

\section{CORONARY ANGIOGRAPHY}

Coronary angiography was performed within three months of dopamine stress echocardiography in 116 patients. Lesions were quantified as previously described. ${ }^{7}$ Significant coronary artery disease was defined as diameter stenosis of $\geqslant 50 \%$ in one or more major epicardial arteries.

\section{STATISTICAL ANALYSIS}

Unless otherwise specified, data are presented as mean (SD). The $\chi^{2}$ test was used to compare differences between proportions. The Student's $t$ test was used for analysis of continuous data. Stepwise logistic regression models were fitted to identify independent predictors of chronotropic incompetence. Differences were considered significant if the null hypothesis could be rejected at the 0.05 probability level.

\section{Results}

HAEMODYNAMIC RESPONSES

The target heart rate was achieved in 316 patients without the need for atropine. The maximum dobutamine dose was $10 \mu \mathrm{g} / \mathrm{kg} / \mathrm{min}$ in four patients, $20 \mu \mathrm{g}$ in 33 patients, $30 \mu \mathrm{g}$ in 89 patients, and $40 \mu \mathrm{g}$ in 386 patients. There were 196 patients who failed to achieve the target heart rate at maximum dobutamine dose. These patients comprised the group with chronotropic incompetence. Atropine was given to all of these patients. The target heart rate was reached in 153 of the 196 patients with chronotropic incompetence (78\%). Thus chronotropic incompetence refractory to atropine was encountered in 43 patients (22\%), representing $8 \%$ of the entire population of the study.

The clinical characteristics, drugs, and indications for stress testing in patients with and without chronotropic incompetence are given in table 1. Patients with chronotropic incompetence were significantly younger, more likely to be men and to smoke cigarettes, and had a higher prevalence of previous myocardial infarction and a lower prevalence of hypertension than patients without chronotropic incompetence.

Atropine induced a significant increase in heart rate in patients with chronotropic incompetence (from 112 (20) to 142 (16) beats/min, $\mathrm{p}<0.0001)$. Haemodynamic data in patients with and without chronotropic incompetence are given in table 2. Patients with chronotropic incompetence had a lower resting and peak dobutamine heart rate, a lower resting systolic and diastolic blood pressure, and received a higher dose of dobutamine.

The prevalence of symptoms was similar in patients with and without chronotropic incompetence (table 3). Premature ventricular 
Table 1 Clinical features of patients with and without chronotropic incompetence during dobutamine stress echocardiography

\begin{tabular}{|c|c|c|c|}
\hline \multirow[b]{2}{*}{ Clinical features } & \multicolumn{2}{|c|}{ Chronotropic incompetence } & \multirow[b]{2}{*}{$p$ Value } \\
\hline & $\begin{array}{l}\text { Yes } \\
(n=196)\end{array}$ & $\begin{array}{l}\text { No } \\
(n=316)\end{array}$ & \\
\hline Age (years) (mean (SD)) & $56(13)$ & $62(11)$ & $<0.00001$ \\
\hline Male & $145(74)$ & $168(53)$ & $<0.00001$ \\
\hline Previous myocardial infarction & $108(55)$ & $131(41)$ & $<0.005$ \\
\hline History of heart failure & $22(11)$ & $52(16)$ & 0.1 \\
\hline History of hypertension & $56(29)$ & $126(40)$ & 0.01 \\
\hline Diabetes mellitus & $27(14)$ & $42(13)$ & 0.9 \\
\hline Smoking & $78(40)$ & $94(30)$ & 0.04 \\
\hline Hypercholesterolaemia & $40(20)$ & $60(19)$ & 0.9 \\
\hline Height $(\mathrm{cm})($ mean $(\mathrm{SD}))$ & $173(9)$ & $169(10)$ & $<0.0005$ \\
\hline Body weight (kg) (mean (SD)) & $75(13)$ & $74(14)$ & 0.5 \\
\hline \multicolumn{4}{|l|}{ Drugs } \\
\hline Dihydropyridine CA & $27(14)$ & $48(15)$ & 0.7 \\
\hline Non-dihydropyridine CA & $28(14)$ & $53(17)$ & 0.5 \\
\hline Nitrates & $63(32)$ & $84(27)$ & 0.8 \\
\hline ACE inhibitors & $52(26)$ & $108(34)$ & 0.07 \\
\hline Diuretics & $34(17)$ & $83(26)$ & 0.02 \\
\hline \multicolumn{4}{|l|}{ Indications for stress testing } \\
\hline Atypical chest pain & $62(32)$ & $130(41)$ & 0.03 \\
\hline Typical chest pain & $58(30)$ & $79(25)$ & 0.07 \\
\hline Exertional dyspnoea or fatigue & $10(5)$ & $18(6)$ & 0.8 \\
\hline $\begin{array}{l}\text { Assessment post-MI or after } \\
\text { revascularisation }\end{array}$ & $66(34)$ & $89(28)$ & 0.2 \\
\hline
\end{tabular}

Values are $\mathrm{n}(\%)$ unless otherwise stated.

$\mathrm{ACE}$, angiotensin converting enzyme; CA, calcium antagonists; MI, myocardial infarction.

Table 2 Haemodynamic data in patients with and without chronotropic incompetence during dobutamine stress echocardiography

\begin{tabular}{|c|c|c|c|}
\hline \multirow[b]{2}{*}{ Haemodynamic and stress test variables } & \multicolumn{2}{|c|}{ Chronotropic incompetence } & \multirow[b]{2}{*}{$p$ Value } \\
\hline & $\begin{array}{l}\text { Yes } \\
(n=196)\end{array}$ & $\begin{array}{l}\text { No } \\
(n=316)\end{array}$ & \\
\hline $\mathrm{HR}$ at rest (beats/min) & $71(12)$ & $78(13)$ & $<0.00001$ \\
\hline HR at peak dobutamine (beats/min) & $112(21)$ & $133(16)$ & $<0.00001$ \\
\hline HR increment from rest to peak dobutamine dose & $41(8)$ & $55(9)$ & $<0.00001$ \\
\hline Systolic $\mathrm{BP}$ at rest $(\mathrm{mm} \mathrm{Hg})$ & $127(21)$ & $137(23)$ & $<0.00001$ \\
\hline Systolic BP at peak dobutamine $(\mathrm{mm} \mathrm{Hg})$ & $135(26)$ & $139(28)$ & 0.3 \\
\hline Diastolic $\mathrm{BP}$ at rest $(\mathrm{mm} \mathrm{Hg})$ & $73(13)$ & $77(13)$ & $<0.005$ \\
\hline Diastolic $\mathrm{BP}$ at peak dobutamine $(\mathrm{mm} \mathrm{Hg})$ & $69(13)$ & $71(15)$ & 0.6 \\
\hline Systolic BP drop $>20 \mathrm{~mm} \mathrm{Hg}$ & $30(15 \%)$ & $59(19 \%)$ & 0.3 \\
\hline Systolic BP drop $>40 \mathrm{~mm} \mathrm{Hg}$ & $9(5 \%)$ & $12(4 \%)$ & 0.7 \\
\hline Maximum dobutamine dose $(\mu \mathrm{g} / \mathrm{kg} / \mathrm{min})$ & 40 & $34.5(7.5)$ & $<0.00001$ \\
\hline Angina during the test & $41(21 \%)$ & $59(19 \%)$ & 0.5 \\
\hline ST segment depression & $30(15 \%)$ & $43(14 \%)$ & 0.6 \\
\hline
\end{tabular}

Values are mean (SD) or $\mathrm{n}(\%)$.

$\mathrm{BP}$, blood pressure; HR, heart rate.

Table 3 Symptoms and arrhythmias in patients with and without chronotropic incompetence during dobutamine stress echocardiography

\begin{tabular}{llll}
\hline & \multicolumn{2}{l}{ Chronotropic } & \\
\cline { 2 - 3 } Symptompetence & \\
\cline { 2 - 3 } & $\begin{array}{l}\text { Yes } \\
(n=196)\end{array}$ & $\begin{array}{l}\text { No } \\
(n=316)\end{array}$ & p Value \\
\hline Nausea & $14(7)$ & $8(3)$ & 0.6 \\
Flushing & $1(0.5)$ & $4(1.2)$ & 0.2 \\
Dizziness & $7(4)$ & $4(1)$ & 0.9 \\
Anxiety & $4(2)$ & $5(1.5)$ & 0.7 \\
Chills & $10(5)$ & $14(4)$ & 1 \\
Headache & $7(4)$ & $17(5)$ & 0.1 \\
Symptomatic hypotension & $1(0.5)$ & $2(0.6)$ & 0.4 \\
Premature atrial contractions & $18(9)$ & $18(6)$ & 0.1 \\
Premature ventricular contractions & $76(39)$ & $91(29)$ & 0.02 \\
Supraventricular tachycardia & $4(2)$ & $11(3)$ & 0.3 \\
Atrial fibrillation & 0 & $3(1)$ & 0.2 \\
Ventricular tachycardia < 10 beats & $7(4)$ & $9(3)$ & 0.6 \\
Ventricular tachycardia $\geqslant 10$ beats & 0 & 0 & \\
\hline
\end{tabular}

Values are $\mathrm{n}(\%)$.
STRESS ECHOCARDIOGRAPHY

Resting wall motion abnormalities were detected in 119 patients with chronotropic incompetence $(61 \%)$ and in $161(51 \%)$ patients without $(p=0.03)$. There was no significant difference between patients with and without chronotropic incompetence with regard to resting wall motion score index (1.39 $(0.49) v 1.38$ (0.49), peak wall motion score index $(1.42(0.53) v 1.41(0.52)$, or the prevalence of ischaemia during the test $(31 \% v$ $28 \%$ ), respectively. Patients with chronotropic incompetence had a trend to a higher prevalence of ischaemia in the posterolateral circulation, comprising the posterior, inferior, and lateral wall $(23 \%$ v $17 \%, \mathrm{p}=0.1)$.

PREVALENCE OF CORONARY ARTERY DISEASE IN PATIENTS WITH AND WITHOUT CHRONOTROPIC INCOMPETENCE

Coronary angiography was performed in 46 patients with and in 70 patients without chronotropic incompetence within three months of dopamine stress echocardiography. There was no significant difference between the two groups with regard to the overall prevalence of coronary artery disease $(76 \% v$ $77 \%, \mathrm{p}=0.9)$, multivessel coronary artery disease $(48 \%$ v $50 \%, \mathrm{p}=0.8)$, right coronary artery disease $(46 \% v 49 \%, \mathrm{p}=0.8)$, or right \pm left circumflex coronary artery disease $(67 \% v$ $61 \%, p=0.5)$. There was a trend to a higher prevalence of left circumflex coronary artery disease in patients with chronotropic incompetence than in those without $(54 \%$ v 39\%, $\mathrm{p}=0.1)$.

\section{INDEPENDENT PREDICTORS OF CHRONOTROPIC}

INCOMPETENCE

By multivariate analysis, independent predictors of chronotropic incompetence were a lower resting heart rate $\left(\mathrm{p}<<0.001, \chi^{2}=29\right)$, younger age $\left(\mathrm{p}<<0.001, \chi^{2}=27\right)$, and male $\operatorname{sex}\left(\mathrm{p}<<0.001, \chi^{2}=15\right)$.

\section{CHRONOTROPIC INCOMPETENCE REFRACTORY TO} ATROPINE

Among the 196 patients with chronotropic incompetence at the maximum dose of dobutamine, 153 achieved the target heart rate after atropine administration (mean dose 0.55 (0.26) $\mathrm{mg}$ ), while 43 (22\%) had chronotropic incompetence refractory to the highest dose of atropine $(1 \mathrm{mg})$. Fourteen of the 43 patients (33\%) who had chronotropic incompetence refractory to atropine had ischaemia during dobutamine stress echocardiography. Therefore the test was considered successful (achievement of the target heart rate or development of ischaemia during dopamine stress echocardiography) in 483 patients (94\%). On multivariate analysis of the characteristics of the 196 patients with chronotropic incompetence, we identified younger age $\left(p<<0.001, \chi^{2}=14\right)$, a slower heart rate at maximum dobutamine dose $(\mathrm{p}<<0.001$, $\left.\chi^{2}=13\right)$, and greater height $\left(\mathrm{p}<0.005, \chi^{2}=7\right)$ as independent predictors of refractory chronotropic incompetence. 
SEX DIFFERENCES

There was no significant difference between men and women with respect to age (60 (12) v 62 (13) years, $\mathrm{p}=0.3$ ) or peak heart rate (121 (22) $v 116$ (20) beats $/ \mathrm{min}, \mathrm{p}=0.2$, respectively). Women received a lower maximum dose of dobutamine than men (34.6 (7.5) v 37.9 (5) $\mu \mathrm{g} / \mathrm{kg} / \mathrm{min}, \mathrm{p}<<0.001)$. In the 386 patients who received the maximum dobutamine dosage $(40 \mu \mathrm{g} / \mathrm{kg} / \mathrm{min})$, chronotropic incompetence was detected in 145 of 263 men $(55 \%)$ and 51 of 123 women $(41 \%)(\mathrm{p}<0.05)$. A target heart rate of 210 - age in women was identified as the cut off point that resulted in an equal prevalence of chronotropic incompetence $(55 \%)$ in men and women.

\section{Discussion}

To our knowledge, this is the first study to evaluate the clinical, haemodynamic, and echocardiographic characteristics of patients with chronotropic incompetence during dobutamine stress echocardiography. Unlike the exercise stress test, ${ }^{1}$ the heart rate response during the dobutamine stress test is independent of the functional capacity of the patient. We included only patients who could complete the standard dobutamine stress protocol or reached the target heart rate. Therefore chronotropic incompetence in this study represents an intrinsic failure to achieve the target heart rate rather than being a result of premature termination of the test. Additionally, we excluded patients receiving $\beta$ blockers to avoid the confounding effect of these drugs on the heart rate response to dobutamine.

PREDICTORS OF CHRONOTROPIC INCOMPETENCE Independent predictors of chronotropic incompetence during dobutamine stress echocardiography using a multivariate analysis model were a lower baseline heart rate, a younger age, and male sex. A lower resting heart rate might have contributed to chronotropic incompetence because in this case a larger increment of heart rate is necessary to achieve the target heart rate. However, patients with chronotropic incompetence also had a lower increment of heart rate from rest to peak dobutamine dose despite receiving a higher dose of dobutamine and despite being younger. One explanation is that patients with chronotropic incompetence had intrinsically lower sinus node activity at rest and in response to dobutamine infusion, though they had no manifest clinical disease of the sinoatrial node. Another explanation is that a higher resting heart rate represents a state of a higher sympathetic than parasympathetic activity or a state of increased sympathetic reactivity of the heart. Therefore patients with a higher baseline heart rate would be more responsive to dobutamine infusion. Supporting this theory is our finding of a higher baseline systolic and diastolic blood pressure in the group without chronotropic incompetence, which is in favour of a higher sympathetic than parasympathetic tone or increased sympathetic responsiveness in patients with than without an adequate chronotropic response.
A younger age was an independent predictor of chronotropic incompetence. The increase in myocardial contractility during dobutamine infusion may be associated reflex activation of parasympathetic tone, which in turn would reduce the chronotropic response to dobutamine, as has been suggested before. ${ }^{16}$ Such an effect may be attenuated in elderly people owing to the reduction in vagal activity that occurs with aging. ${ }^{20}$ Another explanation for the age difference is that the values used to define a target heart rate in relation to age are derived from exercise rather than from a dobutamine stress test. The cardiovascular response to exercise is different from the response to dobutamine infusion, so sex and age related heart rate response to exercise may not necessarily apply to dobutamine. The same factors may explain our finding of a higher prevalence of chronotropic incompetence in men, as the calculated target heart rate for women is less than for men of the same age. ${ }^{12}$

Another factor that may contribute to the age and sex related differences between patients with and without chronotropic incompetence is the difference in drug pharmacokinetics. Healthy elderly subjects have been shown to have increased sensitivity to dobutamine. ${ }^{17}$ In our study, there was no significant difference between men and women with respect to age and maximum dobutamine heart rate despite the fact that women received a significantly smaller dose of dobutamine. This shows that women have a greater heart rate response to dobutamine than men. The presence of age and sex difference between patients with and without chronotropic incompetence warrants a study of the heart rate response to dobutamine in normal individuals related to age and sex to derive a formula for target heart rate during a dobutamine stress test rather than using an arbitrary formula derived from the exercise stress test. In this study, the use of the smaller difference in target heart rate between men and women $(220$ - age in men $v 200$ - age in women) resulted in a similar prevalence of chronotropic incompetence in men and women. However, this analysis only included patients who received the maximum dobutamine dose, to avoid the confounding effect of including women in whom the test was terminated at lower dobutamine dose on achieving the target heart rate defined as 200 - age.

Ischaemia was not predictive of chronotropic incompetence, although previous studies described attenuated baroreceptor sensitivity and reduced efferent cardiac sympathetic activity during experimental acute myocardial ischaemia. ${ }^{21}$ This could be explained by a reduction in the prevalence of ischaemia in patients with impaired chronotropic response, balancing the bradycardia inducing effect of ischaemia. ${ }^{16}$ However, we found a trend to a higher prevalence of ischaemia in the posterolateral circulation in patients with chronotropic incompetence. The greater likelihood of premature ventricular contractions in patients with chronotropic incompetence may be related to the higher prevalence of myocardial 
infarction and wall motion abnormalities in these patients.

CHRONOTROPIC INCOMPETENCE REFRACTORY TO ATROPINE

Refractoriness to atropine occurred in $22 \%$ of patients with chronotropic incompetence at the maximum dobutamine dose. These patients had a lower heart rate at maximum dobutamine dose, which implies that such patients may require more than $1 \mathrm{mg}$ of atropine to achieve the target heart rate. The mean age of these patients was younger, which may be explained by the greater vagal tone in the young and therefore the need for a larger dose of atropine. A greater height was also predictive of refractory chronotropic incompetence. This shows that atropine dose should be adjusted to the body surface area rather than being a fixed value in all patients.

COMPARISON WITH PREVIOUS STUDIES

Attenhofer et al reported paradoxical sinus deceleration during dopamine stress echocardiography in $8 \%$ of patients who underwent coronary angiography. ${ }^{16}$ These patients had a lower overall prevalence of coronary disease and similar prevalence of right coronary artery disease. Sinus node deceleration was attributed in some patients to a vasodepressor reflex. We could not find a significant difference in the overall prevalence of coronary artery disease or right coronary artery disease between patients with and without chronotropic incompetence, although we a found a trend to a higher prevalence of left circumflex coronary artery disease and ischaemia in the posterolateral circulation in patients with chronotropic incompetence. Poldermans et al reported decreased sensitivity to dobutamine in elderly subjects who smoked or had a previous myocardial infarction, ${ }^{17}$ consistent with our findings of a higher prevalence of chronotropic incompetence in smokers and in patients with previous myocardial infarction (though these factors were not independent predictors of chronotropic incompetence in our study). Chuah at al studied the predictors of outcome in 860 patients with known or suspected coronary artery disease who underwent dobutamine stress echocardiography. ${ }^{22}$ In patients with resting wall motion abnormalities there was a trend to a higher prevalence of cardiac events in those who could not achieve the target heart rate compared with those who could $(16.2 \%$ v $8.4 \%, \mathrm{p}=0.07)$. However, patients who did not achieve the target heart rate in that study were more likely to be treated with $\beta$ blockers. This may complicate the interpretation of these results, not only because of the association between $\beta$ blockers and reduced heart rate response to dobutamine but also because of the different clinical characteristics of patients in whom $\beta$ blocker treatment is indicated.

Chronotropic incompetence during exercise stress testing is associated with more severe coronary artery disease and with the presence of coronary artery disease and a poor prognosis. ${ }^{1-5}$ In one report, patients with chronotropic incompetence were found to be older, ${ }^{1}$ in contrast with our findings. This may be explained by withdrawal of the parasympathetic tone during exercise, whereas with dobutamine infusion vagal tone may increase owing to initiation of the Bezold-Jarisch reflex with hypercontractility. ${ }^{16}{ }^{23}$ Such vagal activation may be less evident in older patients because of the known reduction in vagal activity with aging. However, recent work has shown that vagal tone continues to modulate heart rate during exercise. ${ }^{24}$ In the Framingham study, chronotropic incompetence during exercise was detected in $21 \%$ of participants. ${ }^{1}$ In contrast with our findings, patients with chronotropic incompetence were older and had a higher resting blood pressure. In agreement with our findings, however, patients with chronotropic incompetence were more likely to smoke cigarettes and had a lower resting heart rate. However, the Framingham study did not evaluate the influence of sex or left ventricular wall motion abnormalities, as that study did not include women or patients with previous myocardial infraction. Previous studies have shown that patients with heart failure have chronotropic incompetence during exercise. ${ }^{25}$ Although patients with chronotropic incompetence in our study had a higher prevalence of myocardial infarction and resting wall motion abnormalities, there was no difference with respect to a history of congestive heart failure. Heart rate response is a function of exogenous sympathetic stimulation during dobutamine infusion and therefore the response may differ during exercise, as it is dependent on endogenous sympathetic release and responsiveness.

LIMITATIONS OF THE STUDY

Coronary angiography was performed in only $23 \%$ of patients. Therefore, the correlation between chronotropic incompetence and angiographic findings was not established in all patients. This would have been necessary to evaluate the trend towards an increased prevalence of left circumflex coronary artery disease in patients with chronotropic incompetence. Although our study excluded patients receiving $\beta$ blockers, some were receiving vasoactive drugs such as calcium channel blockers, nitrates, and angiotensin converting enzyme inhibitors, which may modify the sympathetic tone and influence the chronotropic response. However, none of these drugs was independently predictive of chronotropic incompetence during dobutamine stress echocardiography. Finally our study evaluated the clinical, haemodynamic, and echocardiographic correlates of chronotropic incompetence, but their prognostic significance remains to be evaluated.

SUMMARY AND CONCLUSIONS

Although chronotropic incompetence during dobutamine stress echocardiography correlated with variables known to be associated with an increased risk of adverse prognosis, such as previous myocardial infarction, smoking, and resting wall motion abnormalities, chronotropic incompetence was associated with other variables linked with a better prognosis, such as younger age, a lower preva- 
lence of hypertension, and a lower resting heart rate. $^{26}$ The relations among sex, age, and chronotropic incompetence show the need to titrate the dobutamine dose on the basis of a specific age and sex related response to a dobutamine infusion rather than on the basis of an exercise stress test. Obtaining these specific heart rate criteria is important in determining whether chronotropic incompetence represents a real failure to achieve a normal response or is the result of applying an inappropriate gold standard.

1 Lauer MS, Okin PM, Larson MG, et al. Impaired heart rate response to graded exercise. Prognostic implications of chronotropic incompetence in the Framingham heart study. Circulation 1996;93:1520-6.

2 Ellestad MH. Chronotropic incompetence. The implication of heart rate response to exercise (compensatory parasymof heart rate response to exercise (compensatory para

pathetic hyperactivity?). Circulation $1996 ; 93: 1485-7$.
3 Brenner SJ, Pashkow FJ, Harvey SA, et al. Chronotropic response to exercise predicts angiographic severity in patients with suspected or stable coronary artery disease. Am $\mathcal{F}$ Cardiol 1995;76:1228-32.

4 Weins RD, Lafia P, Marder CM, et al. Chronotropic incompetence in clinical exercise testing. Am f Cardiol 1984;54 74-8.

5 Ellestad MH, Wan MK. Predictive implications of stress testing: follow-up of 2700 subjects after maximum treadmill stress testing. Circulation 1975;51:363-9.

6 Ruffolo RR. The pharmacology of dobutamine. Am $\mathcal{F}$ Med Sci 1987;294:244-8.

7 Elhendy A, van Domburg RT, Roelandt JRTC, et al. Safety and feasibility of dobutamine-atropine stress testing in
hypertensive patients. Hypertension 1997;29:1232-9.

8 Hepner AM, Bach DS, Armstrong WF. Early chronotropic incompetence predicts the need for atropine during incompetence predicts the need for atropine during
dobutamine stress echocardiography. Am $\mathcal{F}$ Cardiol 1997; dobutamine

9 Elhendy A, Geleijnse ML, Roelandt JRTC, et al. Dobutamine-induced hypoperfusion without transient wall motion abnormalities. Less severe ischemia or less severe stress? f Am Coll Cardiol 1996;27:323-9.

10 Elhendy A, Geleijnse ML, Roelandt JRTC, et al. Assessment of patients after coronary artery bypass grafting by dobutamine stress echocardiography. Am f Cardiol 1996; 77:1234-6.

11 Geleijnse ML, Fioretti PM, Roelandt JRTC. Methodology, feasibility safety and diagnostic accuracy of dobutamine 606 .
12 Sheffield LT. Exercise stress test. In: Braunwald E, ed. Heart disease: a textbook of cardiovascular medicine, 4th ed. disease: a textbook of cardiovascular medici

13 Cornel JH, Balk AHMM, Arnese M, et al. Safety and feasibility of dobutamine-atropine stress echocardiography in patients with ischemic left ventricular dysfunction. F $\mathrm{Am}$ Soc Echocardiogr 1996;9:27-32.

14 Picano E, Mathias W, Pingitore A, et al. Safety and tolerability of dobutamine-atropine stress echocardiography: a prospective multicenter study. Lancet 1994;344: 1190-2.

15 Secknus M, Marwick TH. Evolution of dobutamine echocardiography protocols and indications: safety and side effects in 3011 studies over 5 years. $\mathcal{F} \mathrm{Am}$ Coll Cardiol 1997;29:1234-40.

16 Attenhoffer CH, Pellikka PA, McCully RB, et al. Paradoxical sinus deceleration during dobutamine stress echocardiography: description and angiographic correlation. $\mathcal{F}$ Am Coll Cardiol 1997;29:994-9.

17 Poldermans D, Boersma E, Fioretti PM, et al. Cardiac chronotropic responsiveness to beta-adrenoceptor stimulation is not impaired in the elderly. $\mathcal{f}$ Am Coll Cardiol 1995; 25:995-9.

18 Bellotti P, Fioretti PM, Forster T, et al. Reproducibility of the dobutamine-atropine echocardiography stress test. Echocardiography 1993;10:93-7.

19 Elhendy A, Cornel JH, Roelandt JRTC, et al. Relation between contractile response of akinetic segments during dobutamine stress echocardiography and ischemia assessed by simultaneous 201 thallium SPECT. Am f Cardiol 1996; 77:955-9.

20 Kawamoto A, Shimada K, Matsubayashi K, et al. Cardiovascular regulatory function in elderly patients with hypertension. Hypertension 1989;13:401-7.

21 Hageman GR, Gantenberg NS. Attenuation of baroreceptor changes in cardiac sympathetic efferent activities during acute myocardial ischemia. Am Heart f 1983;126:347-51.

22 Chuah S, Pellikka PA, Roger VL, et al. Role of dobutamine stress echocardiography in predicting outcome in 860 patients with known or suspected coronary artery disease. Circulation 1998;97:1474-80.

23 Mark Al. The Bezold-Jarisch reflex revisited: clinical implications of inhibitory reflexes originating in the heart. $7 \mathrm{Am}$ Coll Cardiol 1983;1:90-102.

24 Marraccini P, Orsini E, Nassi G, et al. Effect of parasympathetic blockade on ischemic threshold in patients with exercise-induced myocardial ischemia. Am f Cardiol 1991; 68:539-42.

25 Colucci WS, Ribeiro J, Rocco MB, et al. Impaired chronotropic response to exercise in patients with congestive heart failure: role of postsynaptic beta adrenergic desensitization. Circulation 1989;2:314-23.

26 Dyer AR, Persky V, Stamler J, et al. Heart rate as a prognostic factor for coronary heart disease and mortality: findings in three Chicago epidemiologic studies. Am $\mathcal{F}$ Epidemiol 1980;112:736-49. 